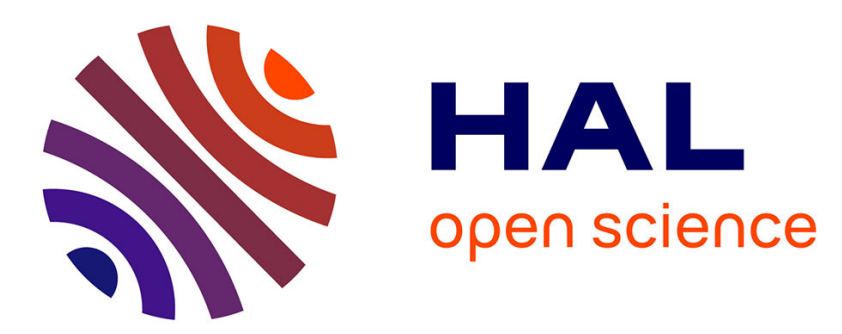

\title{
Fixed-time observer with simple gains for uncertain systems
}

Tomas Menard, Emmanuel Moulay, Wilfrid Perruquetti

\section{To cite this version:}

Tomas Menard, Emmanuel Moulay, Wilfrid Perruquetti. Fixed-time observer with simple gains for uncertain systems. Automatica, 2017, 81, pp.438 - 446. 10.1016/j.automatica.2017.04.009 . hal01516988

\section{HAL Id: hal-01516988 \\ https://hal.science/hal-01516988}

Submitted on 2 May 2017

HAL is a multi-disciplinary open access archive for the deposit and dissemination of scientific research documents, whether they are published or not. The documents may come from teaching and research institutions in France or abroad, or from public or private research centers.
L'archive ouverte pluridisciplinaire HAL, est destinée au dépôt et à la diffusion de documents scientifiques de niveau recherche, publiés ou non, émanant des établissements d'enseignement et de recherche français ou étrangers, des laboratoires publics ou privés. 


\title{
Fixed-time observer with simple gains for uncertain systems
}

\author{
Tomas Ménard ${ }^{\mathrm{a}}$, Emmanuel Moulay ${ }^{\mathrm{b}}$, Wilfrid Perruquetti $^{\mathrm{c}}$ \\ ${ }^{a}$ GREYC, UMR CNRS 6072, Université de Caen, 6 Bd du Maréchal Juin, 14032 Caen Cedex, France. \\ ${ }^{\mathrm{b}}$ XLIM, UMR CNRS 7252, Université de Poitiers, 11 Bvd Marie et Pierre Curie, 86962 futuroscope Chasseneuil, France. \\ ${ }^{\mathrm{c}}$ NON-A, INRIA Lille Nord Europe and CRIStAL UMR CNRS 9189, Ecole Centrale de Lille, 59651 Villeneuve D'Ascq, France.
}

\begin{abstract}
In this article, we consider the problem of fixed-time observer for nonlinear systems, that is a finite-time observer whose settling time can be bounded independently of the initial condition. We consider a large class of nonlinear systems which includes two main classes: linearizable systems up to input-output injection and uniformly observable systems. Furthermore, the effect of noise and uncertainty is analyzed.
\end{abstract}

Key words: high-gain observer; finite-time stability

\section{Introduction}

Observability of nonlinear systems has been characterized within either a differential geometric framework [20] or a differential algebraic framework [13,14]. Once this question is answered for a given system then a practical solution to reconstruct the non measured state has to be worked out. This motivated an increasing number of works on nonlinear observers design during the last decades leading to a great variety of solutions. A first one, which consists in finding a change of coordinates such that the resulting system is linear, has been considered in [12] using algebraic tools. A second one, based on the Lyapunov auxiliary theorem and a direct change of coordinates, can be found in [2]. A third strategy for the class of uniformly observable systems use high-gain observers $[18,15,1]$. Other methods usually rely on a specific structure such as backstepping, adaptive observers, $\mathcal{H}_{\infty}$ observer, etc.

All the above mentioned approaches deal with asymptotic convergence, while finite-time stability has recently become an active area of research [8,9] for the following reasons: better disturbance rejection and robustness

\footnotetext{
* This paper was not presented at any IFAC meeting. Corresponding author T. Ménard.

Email addresses: tomas .menard@unicaen.fr (Tomas

Ménard), emmanuel.moulay@univ-poitiers.fr

(Emmanuel Moulay), wilfrid.perruquetti@ec-lille.fr

(Wilfrid Perruquetti).
}

properties [40] are obtained (because, for system with finite settling-time, the static equivalent gain is "infinite"), this finite-time reponse property is a possible tool for separation principle of nonlinear systems and are well adapt to very severe time response constraint. For example, such property is useful for the synchronization of chaotic signals (with application in secure communication) [32] or for walking robots [33]. Sliding mode observers, which are widely used, allow finite-time convergence, see $[17,31]$, but they are not smooth. Other approaches have been considered in order to obtain finitetime convergence such as moving horizon observers in [29] or delay systems in [28] but they can hardly be generalized to wider classes of nonlinear systems. Quite recently, homogeneity $[9,7]$ was used to obtain finite-time convergence property [9]. Finite-time observers based on recursive construction can be found in $[3,4]$ but the complexity increases with the system's dimension since the corrective term is composed of nested polynomial terms. Another alternative is to design finite-time observers using as simple gains as the one obtained in the linear case. This track has accomplished considerable progress $[32,38,27]$. Other observers with time varying gains or additional assumptions have been developed in $[37,39,25,26]$. In this article, we are interested in a particular kind of stability, namely fixed-time stability. A system is fixed-time stable if it is finite-time stable and if its settling time can be bounded independently of the initial condition. This terminology has been adopted recently in $[34,35]$.

Here we provide fixed-time convergent observers for a 
class of nonlinear systems which includes: the class of linearizable systems up to input-output injection and the class of uniformly observable systems, subject to uncertainty and noise. One of the main advantage of the proposed observer is the simplicity of the gains selection, since they are set off-line by solving the same Riccati equation as in the linear case. Furthermore, a general class of corrective terms for the fixed-time convergence is here proposed, generalizing the existing ones (for finite-time convergence). If there is no uncertainty and no noise, the error is proved to converge in finite-time to the origin. Whereas in the presence of uncertainty and/or noise, the error converges toward a ball whose radius depends on the bound of the noise and/or uncertainty. In both cases, the settling time can be bounded independently of the initial conditions. The two last features are new, indeed, to the authors best knowledge the simplest gains proposed in the literature are those given in $[38,27]$, the first one is only semi-global and the second one does not provide a bound independent of the initial conditions for the settling time. Furthermore the effect of uncertainty and noise has never been studied for this approach.

The article is organized as follows. Section 2, provides some notations and definitions. In section 3 a fixed-time observer is proposed for a large class of nonlinear systems. Then, section 4 gives convergence results in both cases with or without noise/uncertainity. Convincing simulations are given in section 5 . Finally, section 6 concludes the article.

\section{Notations and definitions}

In the paper the following notations are used:

- $\mathbb{R}_{+}=\{x \in \mathbb{R}: x \geq 0\}$ and $\mathbb{R}_{+}^{*}=\{x \in \mathbb{R}: x>0\}$;

- $\mathbb{R}_{+}^{n}=\left(\mathbb{R}_{+}\right)^{n}$, with $n \in \mathbb{N}$;

- $\lceil x\rfloor^{\alpha}=\operatorname{sign}(x) \cdot|x|^{\alpha}$, with $\alpha>0$ and $x \in \mathbb{R}$;

- \|.\| denotes the euclidean norm;

- $\lambda_{m}(M)$ and $\lambda_{M}(M)$ are respectively the lowest and the greatest eigenvalue of the square matrix $M$;

- $\delta_{\lambda}^{r} \triangleq \operatorname{diag}\left(\lambda^{r_{1}}, \ldots, \lambda^{r_{n}}\right)$ for all $r=\left(r_{1}, \ldots, r_{n}\right) \in \mathbb{R}_{+}^{n}$ and $\lambda \in \mathbb{R}_{+}$;

- the function $\nu$ is defined as

$$
\nu(x, \alpha, \beta)=\left\{\begin{array}{lll}
\lceil x\rfloor^{\alpha} & \text { if } & |x|<1 \\
\lceil x\rfloor^{\beta} & \text { if } & |x| \geq 1
\end{array},\right.
$$

with $x \in \mathbb{R}, \alpha \in \mathbb{R}_{+}$and $\beta \in \mathbb{R}_{+}$;

- the matrix $A \in \mathbb{R}^{n \times n}$ and the vectors $B \in \mathbb{R}^{n \times 1}, C \in$
$\mathbb{R}^{1 \times n}$ are defined by

$$
A=\left(\begin{array}{ccccc}
0 & 1 & 0 & 0 & 0 \\
0 & 0 & 1 & 0 & 0 \\
\vdots & \vdots & \vdots & \ddots & \vdots \\
0 & 0 & 0 & 0 & 1 \\
0 & 0 & 0 & 0 & 0
\end{array}\right), B=\left(\begin{array}{c}
0 \\
\vdots \\
0 \\
1
\end{array}\right), C=\left(\begin{array}{c}
1 \\
0 \\
\vdots \\
0
\end{array}\right)^{T}
$$

- $\Delta_{\theta} \triangleq \operatorname{diag}\left(1, \frac{1}{\theta}, \cdots, \frac{1}{\theta^{n-1}}\right)$ with $\theta \geq 1$;

- $F(K, x, \alpha) \triangleq\left(k_{1}\lceil x\rfloor^{\alpha_{1}}, \ldots, k_{n}\lceil x\rfloor^{\alpha_{n}}\right)^{T}$ with $x \quad \in$ $\mathbb{R}, \alpha>0$ and $K=\left(k_{1}, \ldots, k_{n}\right)$;

- a continuous function $\phi: \mathbb{R}^{+} \rightarrow \mathbb{R}^{+}$is said to be of class $\mathcal{K}_{\infty}$ if $\phi$ is strictly increasing, $\phi(0)=0$ and $\lim _{r \rightarrow+\infty} \phi(r)=+\infty$.

One of the key-property used in this article is homogeneity which is defined hereafter.

Definition 1 A function $V: \mathbb{R}^{n} \rightarrow \mathbb{R}$ is homogeneous of degree $d$ with respect to the weights $\left(r_{1}, \ldots, r_{n}\right) \in \mathbb{R}_{+}^{n}$ if $V\left(\delta_{\lambda}^{r} x\right)=\lambda^{d} V(x)$, for all $\lambda>0$ and $x \in \mathbb{R}^{n}$. A vector field $f: \mathbb{R}^{n} \rightarrow \mathbb{R}^{n}$ is homogeneous of degree $d$ with respect to the weights $\left(r_{1}, \ldots, r_{n}\right) \in \mathbb{R}_{+}^{n}$ if for all $1 \leq i \leq n$, the $i$-th component $f_{i}$ is an homogeneous function of degree $_{i}+d$. A dynamical system $\dot{x}=f(x)$ is homogeneous of degree $d$ if the vector field $f$ is homogeneous of degree $d$.

Let $f: \mathbb{R}_{+} \times \mathbb{R}^{n} \rightarrow \mathbb{R}^{n}$ be a continuous vector field, such that $f(0,0)=0$. Consider the system

$$
\left\{\begin{array}{l}
\dot{x}(t)=f(t, x(t)) \\
x(0)=x_{0}
\end{array} .\right.
$$

\section{Definition 2 [35]}

- The equilibrium point $x=0$ of the system (3) is said to be globally finite-time stable if it is globally asymptotically stable and any solution $x(t)$ starting from $x_{0}$ reaches the equilibrium at some finite moment, i.e. $x(t)=0$, for all $t \geq T\left(x_{0}\right)$, where $T: \mathbb{R}^{n} \rightarrow \mathbb{R}^{+}$is the so-called settling time function.

- If in addition the settling-time function $T\left(x_{0}\right)$ is bounded by some positive number $T_{\max }>0$, i.e. $T\left(x_{0}\right) \leq T_{\max }$, for all $x_{0} \in \mathbb{R}^{n}$, the system (3) is said to be globally fixed-time stable.

Remark 1 This last definition can be easily adapted for a compact set instead of an equilibium point.

\section{$3 \quad$ Fixed-time observer design}

When designing observers for nonlinear systems, two main classes of systems are considered: linearizable sys- 
tems up to input-output injections and uniformly observable systems. In the first case, methods for turning systems into a linear system up to input-output injection through diffeomorphism can be found in [23,24,11], and through immersion in $[6,21]$. In the second case of uniformly observable systems, due to the nonlinearity structure of the obtained system, authors use mainly highgain linear corrective term leading to at least a semiglobal exponential convergence of the error (global convergence can be performed when the nonlinearity has a Lipschitz property). Due to the obtained structure different corrective terms can be selected:

- proportional to the output error, which gives exponential convergence,

- proportional to the power of the output error (with power less than one) as in $[32,38]$, which gives semiglobal finite-time convergence,

- a linear combination of the two previous ones, which gives global finite-time convergence.

Here, we will design an observer whose observation error converges to zero in fixed time: for this, a new nonlinear corrective term will be introduced.

\subsection{The class of system under consideration}

We consider the class of uncertain systems which are diffeomorphic to the following triangular form up to inputoutput injection:

$$
\left\{\begin{aligned}
\dot{x}(t) & =A x(t)+\varphi\left(y(t), u(t), \dot{u}(t), \ldots, u^{(r)}(t), x(t)\right) \\
& \quad+B \mathfrak{d}(t) \\
y(t)= & C x(t)+\mathfrak{n}(t)
\end{aligned}\right.
$$

where $x \in \mathbb{R}^{n}$ is the state, $u=\left(u_{1}, \ldots, u_{m}\right) \in \mathbb{R}^{m}$ is the known input (derivatives are known up to order $r$ ), $y \in \mathbb{R}$ is the measured output, $\mathfrak{d} \in \mathbb{R}$ is an unknown exogenous signal (disturbance, modeling error, ....), $\mathfrak{n}$ is the noise on the output and $A, B, C$ are defined by (2). In the rest of the paper the time dependence of the variables will be omitted when it is clear from the context: $x(t)$ will be denoted $x$ and so on. In addition, $\varphi\left(y, u, \dot{u}, \ldots, u^{(r)}, x\right)$ will be denoted as $\varphi(\cdot, x)$ : the $\operatorname{dot}$ means that the function depends on the known variables $y, u, \dot{u}, \ldots, u^{(r)}$. The function $\varphi$ is analytic, with $\varphi(\cdot, 0)=$ 0 and has a triangular structure, that is $\varphi_{i}(\cdot, x)$ only depends on $\cdot, x_{1}, \ldots, x_{i}$. When the function $\varphi$ does not depend on $y$ and the input derivatives (i.e. $\varphi(\cdot, x)$ reduces to $\varphi(u, x)$ ), we recover the triangular form introduced in $[18,19]$ for uniformly observable nonlinear systems. On the contrary, when the function $\varphi$ does not depend on $x$, the model reduces to:

$$
\left\{\begin{array}{l}
\dot{x}=A x+\varphi\left(y, u, \dot{u}, \ldots, u^{(r)}\right)+B \mathfrak{d} \\
y=C x+\mathfrak{n}
\end{array}\right.
$$

and thus, (4) also covers the class of nonlinear system linearizable up to input-output injection.

The design of an observer for the system (4) will be carried out under the following assumptions:

Assumption 1 (nonlinearity) the function $\varphi$ is globally Lipschitz with respect to $x$ with constant $l$;

Assumption 2 (input) all input derivatives are bounded by $u_{0} \in \mathbb{R}_{+}$, that is $\left|u_{i}^{(j)}(t)\right| \leq u_{0}$, for all $t \geq 0$ and $1 \leq i \leq m, 0 \leq j \leq r$;

Assumption 3 (perturbation) the unknown function $\mathfrak{d}$ is essentially bounded, i.e. $\exists \delta_{\mathfrak{d}}>0$; $\operatorname{ess}_{\sup _{t>0}}\|\mathfrak{d}(t)\| \leq$ $\delta_{\mathfrak{d}}$

Assumption 4 (noise) the noise signal $\mathfrak{n}$ is essentially

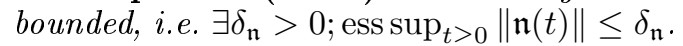

Remark 2 Assumption 1 includes a large class of functions such as $C^{1}$ functions with uniformly bounded derivative. Assumptions 2 to 4 correspond to physical limitations of the different signals, indeed, the noise, uncertainty and the input have finite energy. These assumptions have already been discussed in the literature, since they are the same as the ones used for the classical high-gain observer (see [18,10] for example).

\subsection{Observer and its corrective term}

An observer for the system (4) is given by:

$$
\dot{\hat{x}}=A \hat{x}+\varphi(\cdot, \hat{x})+N^{\alpha, \beta}\left(K, \theta, e_{1}+\mathfrak{n}\right),
$$

and its associated error equation is:

$$
\dot{e}=A e+\Delta \varphi(\cdot, x, \hat{x})+B \mathfrak{d}-N^{\alpha, \beta}\left(K, \theta, e_{1}+\mathfrak{n}\right),
$$

where $\theta \geq 1, \Delta \varphi(\cdot, x, \hat{x})=\varphi(\cdot, x)-\varphi(\cdot, \hat{x})$ and $e_{i}=x_{i}-$ $\hat{x}_{i}, i=1, \ldots, n$, note that $e_{1}+\mathfrak{n}=x_{1}+\mathfrak{n}-\hat{x}_{1}=y-C \hat{x}$. When $\varphi(\cdot, x)$ reduces to $\varphi(u, x))$, by proper selection of $N^{\alpha, \beta}$ we recover the high-gain observer introduced in $[18,19]$ for uniformly observable nonlinear systems. For such high-gain observer the gain $K$ is selected as:

$$
K=\left(k_{1}, \ldots, k_{n}\right) \triangleq S^{-1} C^{T},
$$

with $S$ the symmetric positive definite matrix solution of the Riccati equation:

$$
S+A^{T} S+S A-C^{T} C=0 .
$$

The proof of the error convergence is based on the Lyapunov function:

$$
V(e)=e^{T} S e, \quad e \in \mathbb{R}^{n} .
$$


The corrective term in (6) is chosen in the following form (in order to obtain fixed-time convergence):

$N^{\alpha, \beta}\left(K, \theta, e_{1}\right)=\left(\theta k_{1} \mu^{\alpha_{1}, \beta_{1}}\left(e_{1}\right), \ldots, \theta^{n} k_{n} \mu^{\alpha_{n}, \beta_{n}}\left(e_{1}\right)\right)^{T}$

where the powers $\alpha_{i}, \beta_{i}$ will be specified later on and $\mu^{\alpha, \beta}: \mathbb{R} \rightarrow \mathbb{R}$ is such that the following assumption holds:

\section{Assumption 5 (corrective term)}

(i) for every compact set $C \subset \mathbb{R}^{n} \backslash\{0\}$ and for all $\epsilon>0$, there exists $\lambda_{0}>0, \lambda_{\infty}>0$ such that for all $\lambda \in\left(0, \lambda_{0}\right]$ we have $\max _{x \in C}\left|\frac{\mu^{\alpha, \beta}(\lambda x)}{\lambda^{\alpha}}-\mu^{\alpha, \beta}(x)\right| \leq$ $\epsilon$ and for all $\lambda \in\left[\lambda_{\infty},+\infty\right)$ we have $\max _{x \in C}$ $\left|\frac{\mu^{\alpha, \beta}(\lambda x)}{\lambda^{\beta}}-\mu^{\alpha, \beta}(x)\right| \leq \epsilon ;$

(ii) for every compact set $C \subset \mathbb{R}^{n} \backslash\{0\}$ and for all $\epsilon>$ 0 , there exists $\beta_{M}>1>\alpha_{m}>0$ and $\gamma \geq 1$ such that for all $\alpha \in] \alpha_{m}, 1[, \beta \in] 1, \beta_{M}[$ we have $\max _{x \in C}\left|\mu^{\alpha, \beta}(\lambda x)-\gamma x\right| \leq \epsilon$;

(iii) for all $\epsilon>0, \delta>0$, there exist class $\mathcal{K}_{\infty}$ functions $\tau_{1}, \tau_{2}$ such that for all $\left.x \in \mathbb{R}, z \in[-\delta, \delta], \alpha \in\right] 1-\epsilon[$, $\beta \in] 1,1+\epsilon[$, the following inequality holds

$$
\left|\mu^{\alpha, \beta}(x)-\mu^{\alpha, \beta}(x+z)\right| \leq \tau_{1}(\delta)|x|^{\beta-1}+\tau_{2}(\delta) .
$$

Remark 3 Condition (i) corresponds to the homogeneity in the bi-limit of the function $\mu^{\alpha, \beta}$ (see [3]), it should be noted that the bounds $\lambda_{0}$ and $\lambda_{\infty}$ depend on the compact set $C$ and $\epsilon$. Condition (ii) means that the behavior of the corrective term is close to a linear behavior when $\alpha, \beta$ are close to 1 . Condition (iii) is a kind of weak Lipschitz property which will be useful for the convergence analysis in presence of noise/perturbation.

Example 1 There are several possible corrective terms which verify assumption $\mathbf{5}$ such as:

$\mu^{\alpha, \beta}(x)=-\lceil x\rfloor^{\alpha}-\lceil x\rfloor^{\beta}$,

$\mu^{\alpha, \beta}(x)=-\operatorname{sign}(x)\left(|x|^{\frac{\alpha}{k}}+|x|^{\frac{\beta}{k}}\right)^{k}, \quad k>0$,

$\mu^{\alpha, \beta}(x)=\left\{\begin{array}{l}-\lceil x\rfloor^{\alpha} \text { if } x \leq 1 \\ -\lceil x\rfloor^{\beta} \text { if } x>1\end{array}\right.$.

\section{Observer fixed-time convergence}

In the design of observer (6) we have introduced a bi-limit homogeneous corrective term (11) in order to obtain fixed-time convergence of the observation error. When $\Delta \varphi(\cdot, x, \hat{x})=0$, approximation at 0 or at $\infty$ of $(7)$ is closely related to the behavior of the following system:

$$
\left\{\begin{array}{l}
\dot{e}_{1}=e_{2}-k_{1}\left\lceil e_{1}\right\rfloor^{\alpha_{1}} \\
\vdots \\
\dot{e}_{n-1}=e_{n}-k_{n-1}\left\lceil e_{1}\right\rfloor^{\alpha_{n-1}} \\
\dot{e}_{n}=-k_{n}\left\lceil e_{1}\right\rfloor^{\alpha_{n}}
\end{array},\right.
$$

where $K$ is selected according to (8) and the powers $\alpha_{i}$ are given by

$$
\left.\alpha_{i}=i \alpha-(i-1), \alpha \in\right] 1-1 / n,+\infty[, i=1, \ldots, n .
$$

With such powers, system (15) is homogeneous of degree $(\alpha-1)$ with respect to the weights $r(\alpha)=$ $\left(r_{1}(\alpha), \ldots, r_{n}(\alpha)\right)$ defined by $r_{i}(\alpha)=(i-1) \alpha-(i-$ $2), \quad i=1, \ldots, n$. There exist several methods for constructing an homogeneous Lyapunov function for the system (15) such as in [36]. But here we use the key fact that the 1-level set of the homogeneous Lyapunov function is exactly the 1-level set of the quadratic Lyapunov function used in the linear case. Using this, one can shows (all proofs are postponed to appendices):

Theorem 1 There exists $\epsilon>0$ such that for all $\alpha \in$ ] $1-\epsilon, 1+\epsilon[$, the system (15) is asymptotically stable. Furthermore, an homogeneous Lyapunov function, of degree 2 with respect to the weights $\left(r_{1}(\alpha), \ldots, r_{n}(\alpha)\right)$, for the system (15), whose 1 level set is exactly $\mathcal{H} \triangleq\{e \in$ $\left.\mathbb{R}^{n} \mid e^{T} S e=1\right\}$, is given by

$$
W_{\alpha}\left(\delta_{\lambda}^{r(\alpha)} \tilde{z}\right)=\lambda^{2} W_{\alpha}(\tilde{z})=\lambda^{2} V(\tilde{z})
$$

for all $\lambda>0, \tilde{z} \in \mathcal{H}$. The derivative of the Lyapunov function along the solutions of the system (15) verifies the following inequality

$$
\dot{W}_{\alpha}(e)_{\mid(15)}=\left\langle\nabla W_{\alpha}(e), f_{\alpha}(e)\right\rangle \leq-\frac{3}{4}\left(W_{\alpha}(e)\right)^{\frac{1+\alpha}{2}} .
$$

Where $f_{\alpha}(e)$ denotes the right-hand side of (15). Using such a construction for the Lyapunov function we can build two Lyapunov functions (one for each approximation): at 0 (resp. $\infty$ ) denoted by $W_{\alpha}, \alpha<1$ (resp. $\left.W_{\beta}, \beta>1\right)$. Due to approximations of $N^{\alpha, \beta}\left(K, \theta, e_{1}\right)$ at 0 and at $\infty$, and the fact that we aim at getting fixedtime error convergence, the "powers" $\alpha_{i}, \beta_{i}$ in (11) have to be selected according to (16) with $\alpha \in(1-1 / n, 1)$ and $\beta>1$. Let us denote

$$
\begin{aligned}
\mathcal{H}_{0} & \left.\left.=\left\{\delta_{\lambda}^{r(\alpha)} \tilde{z} \mid \lambda \in\right] 0,1\right], \tilde{z} \in \mathcal{H}\right\}, \\
\mathcal{H}_{\infty} & =\left\{\delta_{\lambda}^{r(\beta)} \tilde{z} \mid \lambda \in[1,+\infty[, \tilde{z} \in \mathcal{H}\} .\right.
\end{aligned}
$$

Note that the sets $\mathcal{H}_{0}$ and $\mathcal{H}_{\infty}$ are equal to $\{e \in$ $\left.\mathbb{R}^{n} \mid e^{T} S e \leq 1\right\}$ and $\left\{e \in \mathbb{R}^{n} \mid e^{T} S e \geq 1\right\}$, respectively. 
Then, the candidate Lyapunov function is defined as:

$$
W_{\alpha, \beta}(e)=\left\{\begin{array}{l}
W_{\alpha}(e) \text { if } e \in \mathcal{H}_{0} \\
W_{\beta}(e) \text { if } e \in \mathcal{H}_{\infty}
\end{array} .\right.
$$

Using this constructed Lyapunov function, we obtain:

Theorem 2 Assume that Assumptions 1 to 5 hold. Let $K$ be given by (8), then there exist $\epsilon>0$ and $\theta^{*} \geq 1$ such that for all $\left.\theta>\theta^{*}, \alpha \in\right] 1-\epsilon, 1[$ and $\beta \in] 1,1+\epsilon[$, the following holds:

- when $\mathfrak{d}=\mathfrak{n}=0$, the origin of the error system (7) is globally fixed-time stable, its settling time $T\left(e_{0}\right)$, where $e_{0}=x(0)-\hat{x}(0)$, is bounded by

$$
T\left(e_{0}\right) \leq \frac{4}{\theta}\left(\frac{1}{1-\alpha}+\frac{1}{\beta-1}\right)
$$

- otherwise there exists a neighborhood of the origin of the error system (7) wich is globally fixed-time stable. More precisely, we have

$$
W_{\alpha, \beta}(e(t)) \leq\left(\frac{c}{\theta} \delta_{\mathfrak{d}}+\theta^{n-1} \tau\left(\delta_{\mathfrak{n}}\right)\right)^{2}+\Gamma e^{-k\left(t-T^{*}\right)}
$$

for all $t \geq T^{*}, e \in \mathbb{R}^{n}$, where $W_{\alpha, \beta}$ is defined by (20), $k, \Gamma, T^{*}, c>0$ are constants and $\tau$ is a class $\mathcal{K}^{\infty}$ function all independent on the initial error $e(0)$, furthermore $c$ and $\tau$ are both independent on $\theta$.

Remark 4 Inequality (22) shows that the error due to the uncertainty will decrease as $\theta$ increases, but in the mean time, the error due to the noise will increase. Hence, there exists an optimal value for $\theta$ such that the overall error is minimum.

This result can be adapted for the linearizable system case and assumptions 1-2 are no more needed:

Corollary 1 Assume that Assumptions 3 to 5 hold. An observer for the system (5) (when $\varphi(\cdot, x)$ is independent of $x$ ) is given by (6) with $\theta=1$, which reads as:

$$
\dot{\hat{x}}=A \hat{x}+\varphi\left(y, u, \dot{u}, \ldots, u^{(r)}\right)+N^{\alpha, \beta}\left(K, 1, e_{1}+\mathfrak{n}\right) .
$$

Let $K$ be given by (8), then there exists $\epsilon>0$ such that for all $\alpha \in] 1-\epsilon, 1[, \beta \in] 1,1+\epsilon[$, the following holds:

- when $\mathfrak{d}=\mathfrak{n}=0$, the origin of the error system is globally fixed-time stable, its settling time $T\left(e_{0}\right)$, where $e_{0}=x(0)-\hat{x}(0)$, is bounded by

$$
T\left(e_{0}\right) \leq 2\left(\frac{1}{1-\alpha}+\frac{1}{\beta-1}\right) .
$$

- otherwise there exists a neighborhood of the origin of the error system wich is globally fixed-time stable. More precisely, we have

$$
W_{\alpha, \beta}(e(t)) \leq\left(c \delta_{\mathfrak{d}}+\tau\left(\delta_{\mathfrak{n}}\right)\right)^{2}+\Gamma e^{-k\left(t-T^{*}\right)}
$$

for all $t \geq T^{*}$, where $c, k, T^{*}>0$ are constants and $\tau$ a class $\mathcal{K}_{\infty}$ function, all independent on the initial error $e(0)$.

Remark 5 Note that the bound (23) on the settling time is not obtained directly from the bound (21) of Theorem 2 by setting $\theta=1$. Indeed, a different over-valuation of the derivative of the Lyapunov function can be obtained since the nonlinear function $\varphi$ in system (5) does not depend on the state $x$.

\section{Simulations}

Let us consider the following system:

$$
\left\{\begin{array}{l}
\dot{x}_{1}=x_{2}-\sin \left(x_{1}\right) \\
\dot{x}_{2}=x_{3}-x_{1}+\sin \left(x_{1}+x_{2}\right) \\
\dot{x}_{3}=-\sin \left(x_{1}+x_{2}+x_{3}\right)+\mathfrak{d} \\
y=x_{1}+\mathfrak{n}
\end{array}\right.
$$

where $\mathfrak{d}=10$ and $\mathfrak{n}$ is a white noise of variance $\sigma^{2}=2$ with zero mean. The observer given by (6) with $\mu^{\alpha, \beta}(x)=\nu(x, \alpha, \beta)$ can be written as:

$\left\{\begin{array}{l}\dot{\hat{x}}_{1}=\hat{x}_{2}-\sin \left(\hat{x}_{1}\right)+\theta k_{1} \nu\left(x_{1}-\hat{x}_{1}, \alpha_{1}, \beta_{1}\right) \\ \dot{\hat{x}}_{2}=\hat{x}_{3}-\hat{x}_{1}+\sin \left(\hat{x}_{1}+\hat{x}_{2}\right)+\theta^{2} k_{2} \nu\left(x_{1}-\hat{x}_{1}, \alpha_{2}, \beta_{2}\right) \\ \dot{\hat{x}}_{3}=-\sin \left(\hat{x}_{1}+\hat{x}_{2}+\hat{x}_{3}\right)+\theta^{3} k_{3} \nu\left(x_{1}-\hat{x}_{1}, \alpha_{3}, \beta_{3}\right)\end{array}\right.$

The gain is given by $K=(3,3,1)$ and the powers are chosen as $\alpha=0.99$ and $\beta=1.2$.

Two sets of simulations are given. The first one illustrates the effect of the noise and uncertainty on the domain of convergence and is reported on Figure 1. For these simulations, the initial conditions are chosen as $x(0)=[10 ;-100 ; 100]^{T}$ and $\hat{x}(0)=[0 ; 0 ; 0]^{T}$. More precisely, only the observation error for $x_{3}$ is reported, since this component is the most affected by the noise and uncertainty. As stated in Theorem 2, we can see that the error due to the uncertainty decreases as $\theta$ increases, but the error due to the noise increases while $\theta$ increases. The optimal value of $\theta$ is comprised between 4 and 5 . The second one illustrates the fixed-time property of the proposed observer and is reported on Figure 2. In this case, the proposed observer is compared with the classical high-gain observer from [18] and the finite-time observer from [38], the tunning parameter is set as $\theta=3$ for every observer. 


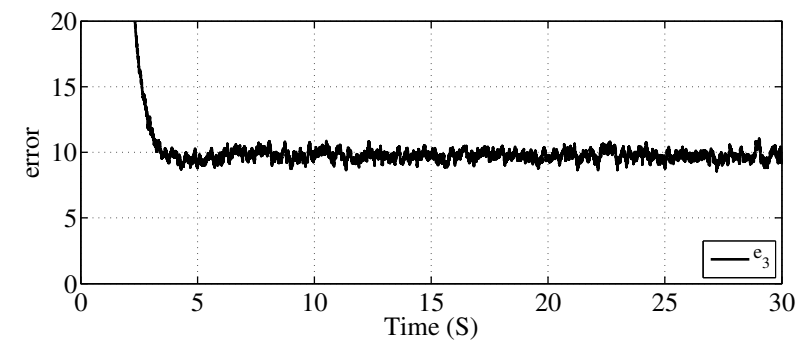

(a) $\theta=3$

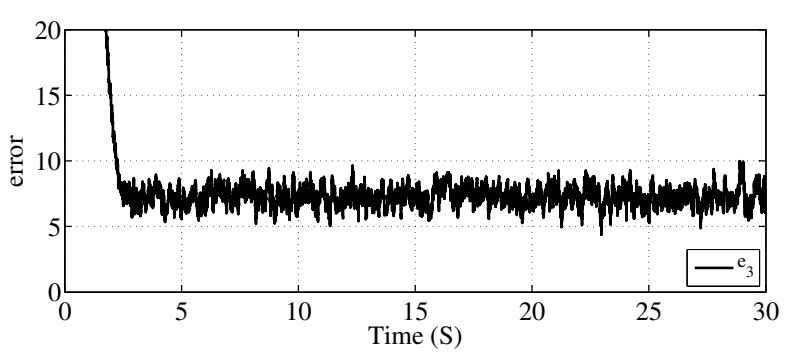

(b) $\theta=4$

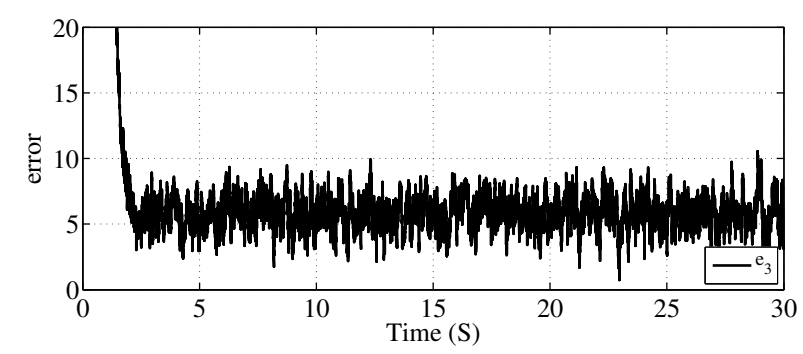

(c) $\theta=5$

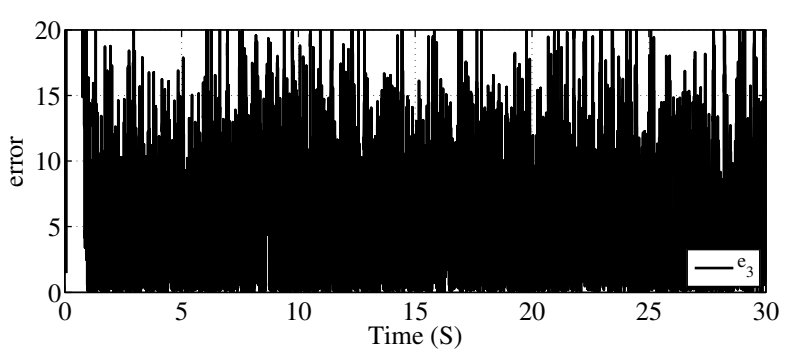

(d) $\theta=10$

Fig. 1. Comparison of the error $e_{3}=\left|x_{3}-\hat{x}_{3}\right|$ for different values of $\theta$.

\section{Conclusion}

In this article, we have proposed a finite-time observer with bounded settling time for a general class of nonlinear systems subject to noise and uncertainty. This class of systems includes the class of linearizable systems up to input-output injection and the class of uniformly observable systems. The gains of this observer are exactly the same than for the classical high-gain observer, which makes it easy to tune. Furthermore, we have shown that

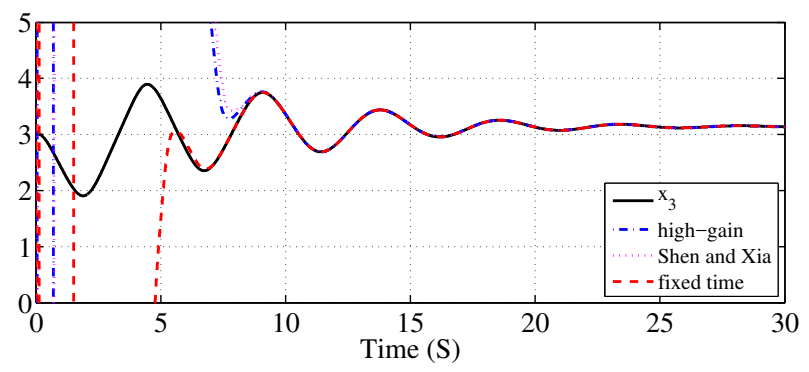

(a) $x(0)=[1,2,3]$ and $\hat{x}(0)=\left[10^{5}, 10^{5}, 10^{5}\right]$

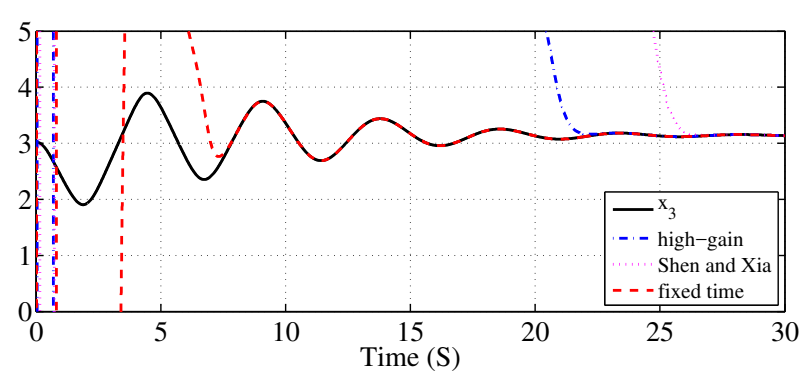

(b) $x(0)=[1,2,3]$ and $\hat{x}(0)=\left[10^{15}, 10^{15}, 10^{15}\right]$

Fig. 2. Comparison of the time of convergence for different initial conditions.

the error converges, in fixed time, toward zero if there are no noise and uncertainty and toward a ball whose radius depends on the noise and the uncertainty bound otherwise.

\section{Acknowledgments}

This work was partially supported by ANR 15 CE23 0007 (Project Finite4SoS).

\section{A Technical Lemmas}

The following technical lemmas will be used throughout the appendices.

Lemma 1 [30, Lemma 26.8] Consider the product space $X \times Y$ where $Y$ is compact. If $N$ is an open subset of $X \times Y$ containing the slice $\left\{x_{0}\right\} \times Y$ of $X \times Y$, then $N$ contains some tube $W \times Y$ about $\left\{x_{0}\right\} \times Y$, where $W$ is a neighborhood of $x_{0}$ in $X$.

Lemma 2 Let $\epsilon>0$ and

$$
\begin{aligned}
g: \mathbb{R}_{+} \times[1-\epsilon, 1+\epsilon] & \rightarrow \mathbb{R}_{+} \\
(x, y) & \mapsto g(x, y)
\end{aligned}
$$

be a $C^{1}$ function on $\left.\mathbb{R}_{+}^{*} \times\right] 1-\epsilon, 1+\epsilon[$ verifying the following assumptions:

i) $\lim _{x \rightarrow 0} g(x, y)=0$, uniformly with respect to $y$; 
ii) $\lim _{x \rightarrow+\infty} g(x, y)=+\infty$, uniformly with respect to $y$

iii) $\left\{x \in \mathbb{R}_{+} \mid g(x, 1)=1\right\}=\{1\}$;

iv) $g(1, y)=1$ for all $y \in[1-\epsilon, 1+\epsilon]$;

v) $\frac{\partial g}{\partial x}(1,1) \neq 0$.

Then, there exists $\epsilon_{*}>0$ such that for every $\left.y \in\right] 1-$ $\epsilon_{*}, 1+\epsilon_{*}[$ we have

$$
\left\{x \in \mathbb{R}_{+} \mid g(x, y)=1\right\}=\{1\}
$$

Proof (by contradiction) Assume that for all $\tilde{\epsilon}>0$ there exists $\tilde{y} \in] 1-\tilde{\epsilon}, 1+\tilde{\epsilon}[$ and $\tilde{x} \neq 1$ such that $g(\tilde{x}, \tilde{y})=1$. On one hand, one can construct a sequence $\left(x_{k}, y_{k}\right)$ such that $y_{k}$ converges toward 1 and such that $g\left(x_{k}, y_{k}\right)=1$ and $x_{k} \neq 1$ for all $k \in \mathbb{N}$. Because of assumptions $i$ ) and ii), there exists $\varsigma>0$ such that one can extract a subsequence of $x_{k}$ contained in $\left[\varsigma, \frac{1}{\varsigma}\right]$. Since this subsequence is contained in the compact set $\left[\varsigma, \frac{1}{\varsigma}\right]$, one can further extract a convergent subsequence $\tilde{x}_{k}$ converging toward 1 because of assumption iii) and the continuity of $g$.

On the other hand, applying the implicit function theorem given for instance in [5, Th. 13.7] to $g$ by using assumption $v$ ) shows that there is a neighborhood of 1 such that for every $y$ in this neighborhood, the equation $g(x, y)=1$ possesses only one solution. By assumption $i v)$, this solution is given by $(1, y)$, which contradicts the existence of the previously constructed subsequence.

Lemma 3 Consider the following ordinary differential equation

$$
\left\{\begin{array}{l}
\dot{x}(t)=-k_{1} \nu\left(x(t), \gamma_{1}, \gamma_{2}\right)+k_{2} \nu\left(x(t), \gamma_{3}, \gamma_{4}\right) \\
x(0)>0
\end{array}\right.
$$

with $k_{1}, k_{2}>0, \gamma_{1}<1, \gamma_{2}>1, \gamma_{1}>\gamma_{3}>0$ and $\gamma_{2}>\gamma_{4}>0$. Then, there exist constants $T, k_{3}, \Gamma>0$ independent of $x(0)$ such that

$$
x(t) \leq \nu\left(\frac{k_{2}}{k_{1}}, \frac{1}{\gamma_{1}-\gamma_{3}}, \frac{1}{\gamma_{2}-\gamma_{4}}\right)+\Gamma e^{-k_{3}(t-T)}
$$

for all $t \geq T$.

Proof One shall prove (A.4) when $\frac{k_{2}}{k_{1}}<1$ (the case $\frac{k_{2}}{k_{1}} \geq 1$ being similar is omited). Let us show firstly that after a time $T>0$, the solution $x(t)$ of (A.3) verifies $x(t)<1$ for any $x(0)>0$, then, for $x(0)<1$ one shows that the equilibrium point $\left(\frac{k_{2}}{k_{1}}\right)^{\frac{1}{\gamma_{1}-\gamma_{3}}}$ is exponentially stable. For $x(t)$, one has $\dot{x}(t) \leq-\left(k_{1}-k_{2}\right) x(t)^{\gamma_{2}}$ and by using the comparison lemma 2.5 p. 85 in [22], one obtains that $x(t)<1$ for $t>T \triangleq \frac{1}{\left(\gamma_{2}-1\right)\left(k_{1}-k_{2}\right)}$.
For $\left(\frac{k_{2}}{k_{1}}\right)^{\frac{1}{\gamma_{1}-\gamma_{3}}} \leq x(t)<1$, one has $\dot{x}(t)=-k_{1} x(t)^{\gamma_{1}}+$ $k_{2} x(t)^{\gamma_{3}} \leq-k_{3}\left(x(t)-\left(\frac{k_{2}}{k_{1}}\right)^{\frac{1}{\gamma_{1}-\gamma_{3}}}\right)$, with $k_{3}=$ $k_{1} \frac{1-\left(\frac{k_{2}}{k_{1}}\right)^{\frac{\gamma_{1}}{\gamma_{1}-\gamma_{3}}}}{1-\left(\frac{k_{2}}{k_{1}}\right)^{\frac{1}{\gamma_{1}-\gamma_{3}}}}+\gamma_{3} k_{2}\left(\frac{k_{2}}{k_{1}}\right)^{\frac{\gamma_{3}-1}{\gamma_{1}-\gamma_{3}}}$. One concludes that for $t \geq T$

$$
x(t) \leq\left(1-\left(\frac{k_{2}}{k_{1}}\right)^{\frac{1}{\gamma_{1}-\gamma_{3}}}\right) e^{-k_{3}(t-T)}+\left(\frac{k_{2}}{k_{1}}\right)^{\frac{1}{\gamma_{1}-\gamma_{3}}} .
$$

\section{B Proof of Theorem 1}

The proof is split into two parts. We first show that there exists $\epsilon_{1}>0$ such that for all $\left.\alpha \in\right] 1-\epsilon_{1}, 1+\epsilon_{1}[$, the function $W_{\alpha}$ is well defined and is a candidate Lyapunov function. Then we show that there exists $0<\epsilon_{2} \leq \epsilon_{1}$ such that for all $\alpha \in] 1-\epsilon_{2}, 1+\epsilon_{2}[$, inequality $(1 \overline{8})$ is satisfied. The asymptotic stability of the system (15) follows from these properties.

\section{Part 1}

In order for (17) to be consistent, we need to check that for each $\tilde{z} \in \mathcal{H}$, the homogeneous ray $\left\{\delta_{\lambda}^{r(\alpha)} \tilde{z} \mid \lambda>0\right\}$ cross the manifold $\mathcal{H}$ only once for $\lambda=1$.

Let $\tilde{z} \in \mathcal{H}$, applying Lemma 2 to the function $(\lambda, \alpha) \rightarrow$ $V\left(\delta_{\lambda}^{r(\alpha)} \tilde{z}\right)$ gives the existence of $\epsilon_{\tilde{z}}>0$ such that for all $\alpha \in] 1-\epsilon_{\tilde{z}}, 1+\epsilon_{\tilde{z}}\left[,\left\{\lambda \in \mathbb{R}_{+} \mid V\left(\delta_{\lambda}^{r(\alpha)} \tilde{z}\right)=1\right\}=\{1\}\right.$.

Since $\mathcal{H}$ is a compact set, there exists $\epsilon_{1}>0$ such that for all $\tilde{z} \in \mathcal{H}$ and $\alpha \in] 1-\epsilon_{1}, 1+\epsilon_{1}[$, one has $\{\lambda \in$ $\left.\mathbb{R}_{+} \mid V\left(\delta_{\lambda}^{r(\alpha)} \tilde{z}\right)=1\right\}=\{1\}$.

Now that the function $W_{\alpha}$ is well defined by (17), it remains to prove that it is actually a Lyapunov function for the system (15). It is clear from its definition (17) that $W_{\alpha}$ is smooth and radially unbounded, since $V$ is a Lyapunov function for the system (15) with $\alpha=1$, and that $W_{\alpha}(0)=0$.

\section{Part 2}

We have

$$
\begin{aligned}
\left\langle\nabla W_{\alpha}\left(\delta_{\lambda}^{r(\alpha)} \tilde{z}\right), f_{\alpha}\left(\delta_{\lambda}^{r(\alpha)} \tilde{z}\right)\right\rangle & =\lambda^{1+\alpha}\left\langle\nabla W_{\alpha}(\tilde{z}), f_{\alpha}(\tilde{z})\right\rangle \\
& =\lambda^{1+\alpha}\left\langle\nabla V(\tilde{z}), f_{\alpha}(\tilde{z})\right\rangle
\end{aligned}
$$

for every $\tilde{z} \in \mathcal{H}$.

The function $f_{\alpha}(\tilde{z})$ is continuous relatively to $\alpha$ and $\tilde{z}$, so the function

$$
\begin{aligned}
h:] 1-\epsilon_{1}, 1+\epsilon_{1}[\times \mathcal{H} & \rightarrow \mathbb{R}_{+} \\
(\alpha, \tilde{z}) & \mapsto-\left\langle\nabla V(\tilde{z}), f_{\alpha}(\tilde{z})\right\rangle
\end{aligned}
$$

is also continuous, and $h(1, \tilde{z}) \geq 1$ for all $\tilde{z} \in \mathcal{H}$. Hence $\{1\} \times \mathcal{H} \subset h^{-1}(] 3 / 4,+\infty[)$ which is an open subset since 
$h$ is continuous. Since $\mathcal{H}$ is a compact set, we can apply Lemma 1 and there exists $0<\epsilon_{2} \leq \epsilon_{1}$ such that

$\left.\left\langle\nabla V(\tilde{z}), f_{\alpha}(\tilde{z})\right\rangle \leq-\frac{3}{4}, \quad \forall \tilde{z} \in \mathcal{H}, \quad \forall \alpha \in\right] 1-\epsilon_{2}, 1+\epsilon_{2}[$.

Finally, we obtain

$$
\begin{aligned}
\left\langle\nabla W_{\alpha}\left(\delta_{\lambda}^{r(\alpha)} \tilde{z}\right), f_{\alpha}\left(\delta_{\lambda}^{r(\alpha)} \tilde{z}\right)\right\rangle & \leq-\frac{3}{4} \lambda^{1+\alpha} \\
& \leq-\frac{3}{4}\left(\lambda^{2} W(\tilde{z})\right)^{\frac{1+\alpha}{2}} \\
& \leq-\frac{3}{4}\left(W\left(\delta_{\lambda}^{r(\alpha)} \tilde{z}\right)\right)^{\frac{1+\alpha}{2}}
\end{aligned}
$$

\section{Proof of Theorem 2}

One considers the candidate Lyapunov function $W_{\alpha, \beta}$ given by (20) for $\alpha \in] 1-\epsilon_{1}, 1[, \beta \in] 1,1+\epsilon_{1}\left[\right.$ where $\epsilon_{1}>0$ is given by Theorem 1 . We first state useful Lemmas for the proof of Theorem 2 .

Lemma 4 There exists $\left.\epsilon_{2} \in\right] 0, \epsilon_{1}[$ such that the following inequality is verified

$$
\begin{aligned}
& \left\langle\nabla W_{\alpha, \beta}(e), A e-N^{\alpha, \beta}\left(K, 1, e_{1}\right)\right\rangle \\
& \quad \leq-\frac{1}{2} \nu\left(W_{\alpha, \beta}(e), \frac{1+\alpha}{2}, \frac{1+\beta}{2}\right)
\end{aligned}
$$

for all $\left.e \in \mathbb{R}^{n}, \alpha \in\right] 1-\epsilon_{2}[, \beta \in] 1,1+\epsilon_{2}[$ and $\nu$ is defined by (1).

Proof Inequality (C.1) is equivalent to the two following inequalities:

$$
\begin{gathered}
\left\langle\nabla W_{\alpha}(e), A e-N^{\alpha, \beta}\left(K, 1, e_{1}\right)\right\rangle \\
\leq-\frac{1}{2}\left(W_{\alpha}(e)\right)^{\frac{1+\alpha}{2}}, \forall e \in \mathcal{H}_{0} \\
\left\langle\nabla W_{\beta}(e), A e-N^{\alpha, \beta}\left(K, 1, e_{1}\right)\right\rangle \\
\leq-\frac{1}{2}\left(W_{\beta}(e)\right)^{\frac{1+\beta}{2}}, \forall e \in \mathcal{H}_{\infty}
\end{gathered}
$$

The cases $e \in \mathcal{H}_{0}$ and $e \in \mathcal{H}_{\infty}$ are very similar to demonstrate, then one shall exhibit only the case $e \in \mathcal{H}_{0}$. One has

$$
\begin{aligned}
\left\langle\nabla W_{\alpha}(e), A e-N^{\alpha, \beta}\left(K, 1, e_{1}\right)\right\rangle & \\
=\frac{4}{5} & \left\langle\nabla W_{\alpha}(e), A e-F\left(K, e_{1}, \alpha\right)\right\rangle \\
+ & \left\langle\nabla W_{\alpha}(e), \frac{1}{5} A e-\left(\gamma-\frac{4}{5}\right) F\left(K, e_{1}, \alpha\right)\right\rangle \\
& +\left\langle\nabla W_{\alpha}(e), \gamma F\left(K, e_{1}, \alpha\right)-N^{\alpha, \beta}\left(K, 1, e_{1}\right)\right\rangle \\
\leq- & \frac{3}{5}\left(W_{\alpha}(e)\right)^{\frac{1+\alpha}{2}} \\
& +\left\langle\nabla W_{\alpha}(e), \frac{1}{5} A e-\left(\gamma-\frac{4}{5}\right) F\left(K, e_{1}, \alpha\right)\right\rangle \\
& +\left\langle\nabla W_{\alpha}(e), \gamma F\left(K, e_{1}, \alpha\right)-N^{\alpha, \beta}\left(K, 1, e_{1}\right)\right\rangle
\end{aligned}
$$

for all $e \in \mathcal{H}_{0}$, because of Theorem 1, with $\gamma$ given by Assumption $\mathbf{5}$.

Let us denote $e=\delta_{\lambda}^{r(\alpha)} \tilde{z}$, with $\tilde{z} \in \mathcal{H}$ and $\lambda \in[0,1]$, then

$$
\begin{aligned}
& \left\langle\nabla W_{\alpha}(e), \gamma F\left(K, e_{1}, \alpha\right)-N^{\alpha, \beta}\left(K, 1, e_{1}\right)\right\rangle= \\
& \lambda^{1+\alpha} \sum_{i=1}^{n}\left(\nabla W_{\alpha}(\tilde{z})\right)_{i} k_{i}\left(\frac{\gamma\left\lceil\lambda \tilde{z}_{1}\right\rfloor^{\alpha_{i}}-\mu_{\alpha_{i}}^{\beta_{i}}\left(\lambda \tilde{z}_{1}\right)}{\lambda^{\alpha_{i}}}\right) .
\end{aligned}
$$

According to Assumption 5- $(i)$ the function $\mu_{\alpha}^{\beta}(x)$ converges toward $\gamma\lceil x\rfloor^{\alpha}$ when $\alpha, \beta \rightarrow 1$, uniformly on any compact set. Thus there exists $0<\epsilon_{21}<\epsilon_{1}$ such that

$$
\sum_{i=1}^{n}\left(\nabla W_{\alpha}(\tilde{z})\right)_{i} k_{i}\left(\frac{\gamma\left\lceil\lambda \tilde{z}_{1}\right\rfloor^{\alpha_{i}}-\mu_{\alpha_{i}}^{\beta_{i}}\left(\lambda \tilde{z}_{1}\right)}{\lambda^{\alpha_{i}}}\right) \leq \frac{1}{20 \gamma}
$$

for every $\alpha \in] 1-\epsilon_{21}, 1[, \beta \in] 1,1+\epsilon_{21}[, \tilde{z} \in \mathcal{H}$ and $\lambda \in[0,1]$.

Since $\gamma \geq 1$, one has

$$
\left\langle\nabla W_{1}(e), \frac{1}{5} A e-\left(\gamma-\frac{4}{5}\right) F\left(K, e_{1}, 1\right)\right\rangle<0
$$

for all $e \in \mathcal{H}$. Applying the Lemma 1 gives the existence of $\epsilon_{22}>0$ such that

$$
\left\langle\nabla W_{\alpha}(e), \frac{1}{5} A e-\left(\gamma-\frac{4}{5}\right) F\left(K, e_{1}, \alpha\right)\right\rangle \leq 0
$$

for all $e \in \mathcal{H}$ and $\alpha \in] 1-\epsilon_{22}, 1[$. This inequality can be extended, by homogeneity, for all $e \in \mathcal{H}_{0}$ and $\alpha \in$ $11-\epsilon_{22}, 1[$.

Finally, one obtains

$$
\begin{aligned}
& \left\langle\nabla W_{\alpha}(e), A e-N^{\alpha, \beta}\left(K, 1, e_{1}\right)\right\rangle \\
& \leq-\frac{3}{5}\left(W_{\alpha}(e)\right)^{\frac{1+\alpha}{2}}+\frac{1}{10} \lambda^{1+\alpha} \\
& \leq-\frac{3}{5}\left(W_{\alpha}(e)\right)^{\frac{1+\alpha}{2}}+\frac{1}{10}\left(\lambda^{2} W_{\alpha}(\tilde{z})\right)^{\frac{1+\alpha}{2}} \\
& \leq-\frac{1}{2}\left(W_{\alpha}(e)\right)^{\frac{1+\alpha}{2}}
\end{aligned}
$$


for all $e \in \mathcal{H}_{0}$ and $\left.\alpha \in\right] 1-\epsilon_{2}, 1\left[\right.$, with $\epsilon_{2}=\min \left\{\epsilon_{21}, \epsilon_{22}\right\}$.

Lemma 5 There exists $M>0$ such that for all $\theta \geq 1$

$$
\left\langle\nabla W_{\alpha, \beta}(\bar{e}), \Delta_{\theta} \Delta \varphi(\cdot, x, \hat{x})\right\rangle \leq M W_{\alpha, \beta}(\bar{e})
$$

for all $\alpha \in] 1-\epsilon_{1}, 1[, \beta \in] 1,+\epsilon_{1}\left[\right.$ and $x, \hat{x} \in \mathbb{R}^{n}$, where $e=x-\hat{x}, \bar{e}=\Delta_{\theta} e$.

Proof Two cases have to be considered: $\bar{e} \in \mathcal{H}_{0}$ and $\bar{e} \in \mathcal{H}_{\infty}$, where $\mathcal{H}_{0}$ and $\mathcal{H}_{\infty}$ are defined by (19). Since the two cases are very similar, one only proves the case $\bar{e} \in \mathcal{H}_{0}$. Since $\bar{e} \in \mathcal{H}_{0}$, there exists $\lambda \in[0,1]$ and $\bar{z} \in \mathcal{H}$ such that $\bar{e}=\delta_{\lambda}^{r(\alpha)} \bar{z}$. Let $z, \hat{z}, \tilde{z} \in \mathbb{R}^{n}$ be such that $x=$ $\delta_{\lambda}^{r(\alpha)} z, \hat{x}=\delta_{\lambda}^{r(\alpha)} \hat{z}$ and $e=\delta_{\lambda}^{r(\alpha)} \tilde{z}$ respectively, then

$$
\begin{aligned}
& \left\langle\nabla W_{\alpha, \beta}(\bar{e}), \Delta_{\theta} \Delta \varphi(\cdot, x, \hat{x})\right\rangle \\
= & \lambda^{2}\left\langle\nabla W_{\alpha}(\bar{z}), \delta_{\lambda}^{-r(\alpha)} \Delta_{\theta} \Delta \varphi(\cdot, x, \hat{x})\right\rangle, \\
= & \lambda^{2} \bar{z}^{T} S \delta_{\lambda}^{-r(\alpha)} \Delta_{\theta} \Delta \varphi(\cdot, x, \hat{x}) .
\end{aligned}
$$

Applying the mean value Theorem [5, Th. 12.9], there exists $x_{\xi} \in[x, \hat{x}]$ such that $\lambda^{2} \bar{z}^{T} S \delta_{\lambda}^{-r(\alpha)} \Delta_{\theta} \Delta \varphi(\cdot, x, \hat{x})=$ $\lambda^{2} \bar{z}^{T} S \delta_{\lambda}^{-r(\alpha)} \frac{\partial \varphi\left(\cdot, x_{\xi}\right)}{\partial x}(x-\hat{x})$. Then, we obtain

$$
\begin{aligned}
& \left\langle\nabla W_{\alpha, \beta}(\bar{e}), \Delta_{\theta} \Delta \varphi(\cdot, x, \hat{x})\right\rangle=\left(\lambda \bar{z}^{T}\right) S \\
& \times\left(\delta_{\lambda}^{1-r(\alpha)} \Delta_{\theta} \frac{\partial \varphi\left(\cdot, x_{\xi}\right)}{\partial x} \Delta_{\theta}^{-1} \delta_{\lambda}^{r(\alpha)-1} \delta_{\lambda}^{1-r(\alpha)} \Delta_{\theta}(x-\hat{x})\right) .
\end{aligned}
$$

Given the triangular structure of $\varphi$, and the fact that $1 \geq \lambda^{1-r_{1}(\alpha)}>\cdots>\lambda^{1-r_{n}(\alpha)}$, one can proceed as in [16], which gives the existence of a constant $M>0$ such that

$$
\begin{aligned}
\left\langle\nabla W_{\alpha, \beta}(\bar{e}), \Delta_{\theta} \Delta \varphi(\cdot, x, \hat{x})\right\rangle & \leq M \lambda^{2} \bar{z}^{T} S \bar{z} \\
& \leq M W_{\alpha, \beta}(\bar{e})
\end{aligned}
$$

Note that the constant $M$ depends on the bound on the input and its derivatives $u_{0}$, the Lipschitz constant $l$ of $\varphi$, the dimension of the system $n$, the number of input $m$ and the matrix $S$.

\section{Lemma 6 The following two inequalities hold}

$$
\begin{aligned}
& \left\langle\nabla W_{\alpha, \beta}(\bar{e}), \Delta_{\theta} B \mathfrak{d}(t)\right\rangle \leq \frac{\sqrt{\lambda_{M}(S)} \delta_{\mathfrak{d}}}{\theta^{n-1}} \sqrt{W_{\alpha, \beta}(\bar{e})} \\
& \left\langle\nabla W_{\alpha, \beta}(\bar{e}), N^{\alpha, \beta}\left(K, 1, \bar{e}_{1}\right)-N^{\alpha, \beta}\left(K, 1, \bar{e}_{1}+\mathfrak{n}(t)\right)\right\rangle \\
& \quad \leq \tau_{3}\left(\delta_{\mathfrak{n}}\right) \nu\left(W_{\alpha, \beta}(\bar{e}), \frac{1}{2}, \frac{\beta}{2}\right)
\end{aligned}
$$

for all $\left.\bar{e} \in \mathbb{R}^{n}, \alpha \in\right] 1-\epsilon_{1}, 1[, \beta \in] 1,1+\epsilon_{1}[$ and for almost all $t \geq 0$, where $\tau_{3}$ is a class $\mathcal{K}^{\infty}$ function.
Proof Let us first prove inequality (C.4) for $\bar{e} \in \mathcal{H}_{0}$, the case $\bar{e} \in \mathcal{H}_{\infty}$ is very similar and then left to the reader. Since $\bar{e} \in \mathcal{H}_{0}$, there exists $\lambda \in[0,1]$ and $\bar{z} \in \mathcal{H}$ such that $\bar{e}=\delta_{\lambda}^{r(\alpha)} \bar{z}$. One has

$$
\begin{aligned}
\left\langle\nabla W_{\alpha}(\bar{e}), \Delta_{\theta} B \mathfrak{d}\right\rangle & =\frac{1}{\theta^{n-1}}\left\langle\nabla W_{\alpha}\left(\delta_{\lambda}^{r(\alpha)} \bar{z}\right), B \mathfrak{d}\right\rangle, \\
& =\frac{\lambda^{2-r_{1}(\alpha)}}{\theta^{n-1}}\langle\nabla V(\bar{z}), B \mathfrak{d}\rangle, \\
& \leq \frac{\lambda}{\theta^{n-1}} \sqrt{\overline{z^{T}} S \bar{z}}|\mathfrak{d}| \sqrt{B^{T} S B} \\
& \leq \frac{\sqrt{\lambda_{M}(S)} \delta_{\mathfrak{d}}}{\theta^{n-1}} \sqrt{W_{\alpha}(\bar{e})} .
\end{aligned}
$$

Let us now prove inequality (C.5). Similarly to what has been done previously, we only consider the case $\bar{e} \in \mathcal{H}_{\infty}$. There exists $\bar{z} \in \mathcal{H}$ such that $\bar{e}=\delta_{\lambda}^{r(\beta)} \bar{z}$, with $\lambda \in$ $[1,+\infty[$. It follows that

$$
\begin{aligned}
& \left\langle\nabla W_{\alpha, \beta}(\bar{e}), N^{\alpha, \beta}\left(K, 1, \bar{e}_{1}\right)-N^{\alpha, \beta}\left(K, 1, \bar{e}_{1}+\mathfrak{n}(t)\right)\right\rangle \\
& =\lambda^{2}\left\langle\nabla W_{\beta}(\bar{z}), \delta_{\lambda}^{-r(\beta)}\right. \\
& \left(N^{\alpha, \beta}\left(K, 1, \bar{e}_{1}\right)-N^{\alpha, \beta}\left(K, 1, \bar{e}_{1}+\mathfrak{n}(t)\right)\right\rangle, \\
& \leq \lambda^{2} \sqrt{\lambda_{M}(S)} \sqrt{V(\bar{z})} \\
& \sqrt{\sum_{i=1}^{n} \lambda^{-2 r_{i}(\beta)} k_{i}^{2}\left|\mu_{\alpha_{i}}^{\beta_{i}}\left(\bar{e}_{1}\right)-\mu_{\alpha_{i}}^{\beta_{i}}\left(\bar{e}_{1}+\mathfrak{n}(t)\right)\right|^{2}}, \\
& \leq \lambda^{2} \sqrt{\lambda_{M}(S)} \sqrt{W_{\beta}(\bar{z})} \\
& \sum_{i=1}^{n} \lambda^{-r_{i}(\beta)}\left|k_{i}\right|\left|\mu_{\alpha_{i}}^{\beta_{i}}\left(\bar{e}_{1}\right)-\mu_{\alpha_{i}}^{\beta_{i}}\left(\bar{e}_{1}+\mathfrak{n}(t)\right)\right|, \\
& \leq \sqrt{\lambda_{M}(S)} \sqrt{W_{\beta}(\bar{e})} \\
& \sum_{i=1}^{n} \lambda^{1-r_{i}(\beta)}\left|k_{i}\right|\left(\tau_{1}\left(\delta_{\mathfrak{n}}\right)\left|\bar{e}_{1}\right|^{\beta_{i}-1}+\tau_{2}\left(\delta_{\mathfrak{n}}\right)\right), \\
& \leq \sqrt{\lambda_{M}(S)} \sqrt{W_{\beta}(\bar{e})} \\
& \max _{i=1, \ldots, n}\left|k_{i}\right| \sum_{i=1}^{n} \lambda^{1-r_{i}(\beta)+\beta_{i-1}}\left(\frac{\tau_{1}\left(\delta_{\mathfrak{n}}\right)}{\sqrt{\lambda_{m}(S)}}+\tau_{2}\left(\delta_{\mathfrak{n}}\right)\right), \\
& \leq \sqrt{\lambda_{M}(S)} \sqrt{W_{\beta}(\bar{e})} \\
& \max _{i=1, \ldots, n}\left|k_{i}\right| \sum_{i=1}^{n} \lambda^{\beta-1}\left(\frac{\tau_{1}\left(\delta_{\mathfrak{n}}\right)}{\sqrt{\lambda_{m}(S)}}+\tau_{2}\left(\delta_{\mathfrak{n}}\right)\right), \\
& \leq \sqrt{\lambda_{M}(S)}\left(W_{\beta}(\bar{e})\right)^{\frac{\beta}{2}} \\
& \max _{i=1, \ldots, n}\left|k_{i}\right| n\left(\frac{\tau_{1}\left(\delta_{\mathfrak{n}}\right)}{\sqrt{\lambda_{m}(S)}}+\tau_{2}\left(\delta_{\mathfrak{n}}\right)\right) \text {, } \\
& \triangleq \tau_{3}\left(\delta_{\mathfrak{n}}\right)\left(W_{\beta}(\bar{e})\right)^{\frac{\beta}{2}} \text {, }
\end{aligned}
$$

where $\tau_{1}, \tau_{2}$ are given by Assumption 5-(iii). 


\section{Proof of Theorem 2}

The error dynamics given by (7) is recalled here

$\dot{e}=A e-N^{\alpha, \beta}\left(K, \theta, e_{1}+\mathfrak{n}\right)+\Delta \varphi(\cdot, x, \hat{x})+B \mathfrak{d}$.

Let us denote $\bar{e}=\Delta_{\theta} e$. Since $\Delta_{\theta} A \Delta_{\theta}^{-1}=\theta A, C \Delta_{\theta}^{-1}=$ $C$ and $\Delta_{\theta} N^{\alpha, \beta}\left(K, \theta, e_{1}\right)=\theta N^{\alpha, \beta}\left(K, 1, e_{1}\right)$, one obtains

$$
\begin{aligned}
\dot{\bar{e}}= & \theta A \bar{e}-\theta N^{\alpha, \beta}\left(K, 1, \bar{e}_{1}+\mathfrak{n}\right)+\Delta_{\theta} \Delta \varphi(\cdot, x, \hat{x})+\Delta_{\theta} B \mathfrak{d}, \\
= & \theta\left(A \bar{e}-N^{\alpha, \beta}\left(K, 1, \bar{e}_{1}\right)\right)+\Delta_{\theta} \Delta \varphi(\cdot, x, \hat{x})+\Delta_{\theta} B \mathfrak{d} \\
& +\theta\left(N^{\alpha, \beta}\left(K, 1, \bar{e}_{1}\right)-N^{\alpha, \beta}\left(K, 1, \bar{e}_{1}+\mathfrak{n}\right)\right) . \quad(\text { C.7 })
\end{aligned}
$$

By using Lemmas 4, 5 and 6, one gets the existence of $\epsilon>0$ such that

$$
\begin{aligned}
& \dot{W}_{\alpha, \beta}(\bar{e})_{\mid(C .7)} \leq-\left(\frac{1}{2} \theta-M\right) \nu\left(W_{\alpha, \beta}(\bar{e}), \frac{1+\alpha}{2}, \frac{1+\beta}{2}\right) \\
& +\left(\frac{\sqrt{\lambda_{M}(S)} \delta_{\mathfrak{d}}}{\theta^{n-1}}+\theta \tau_{3}\left(\delta_{\mathfrak{n}}\right)\right) \nu\left(\left(W_{\alpha, \beta}(\bar{e}), \frac{1}{2}, \frac{\beta}{2}\right) .\right.
\end{aligned}
$$

for all $\left.\bar{e} \in \mathbb{R}^{n}, \alpha \in\right] 1-\epsilon, 1[, \beta \in] 1,1+\epsilon[$ and $\theta \geq 1$. Then there exists $\theta^{*}>1$ such that for all $\theta>\theta^{*}$

$$
\begin{aligned}
& \dot{W}_{\alpha, \beta}(\bar{e})_{\mid(C .7)} \leq-\frac{1}{4} \theta \nu\left(W_{\alpha, \beta}(\bar{e}), \frac{1+\alpha}{2}, \frac{1+\beta}{2}\right) \\
& +\left(\frac{\sqrt{\lambda_{M}(S)} \delta_{\mathfrak{d}}}{\theta^{n-1}}+\theta \tau_{3}\left(\delta_{\mathfrak{n}}\right)\right) \nu\left(\left(W_{\alpha, \beta}(\bar{e}), \frac{1}{2}, \frac{\beta}{2}\right)\right.
\end{aligned}
$$

If $\delta_{\mathfrak{n}}, \delta_{\mathfrak{d}}=0$, then inequality (C.8) reads as

$$
\begin{aligned}
& \dot{W}_{\alpha, \beta}(\bar{e})_{\mid(C .7)} \leq-\frac{1}{4} \theta\left(W_{\alpha}(\bar{e})\right)^{\frac{1+\alpha}{2}}, \forall \bar{e} \in \mathcal{H}_{0} \\
& \dot{W}_{\alpha, \beta}(\bar{e})_{\mid(C .7)} \leq-\frac{1}{4} \theta\left(W_{\beta}(\bar{e})\right)^{\frac{1+\beta}{2}}, \forall \bar{e} \in \mathcal{H}_{\infty} .
\end{aligned}
$$

Inequality (C.10) ensures that for any initial condition $\bar{e} \in \mathbb{R}^{n}$, the error trajectory $\bar{e}(t)$ enters $\mathcal{H}_{0}$ after a finitetime $\frac{4}{\theta(\beta-1)}$, while inequality (C.9) ensures that any trajectory $\bar{e}(t)$ belonging to $\mathcal{H}_{0}$, at time $t$, will reach the origin after time $t+\frac{4}{\theta(1-\alpha)}$.

If $\delta_{\mathfrak{n}}, \delta_{\mathfrak{d}} \neq 0$, applying Lemma 3 , gives the existence of $k, \Gamma_{1}, T^{*}$ independent of $\bar{e}(0)$ such that

$$
W_{\alpha, \beta}(\bar{e}) \leq \nu\left(4 \frac{\sqrt{\lambda_{M}(S)}}{\theta^{n}} \delta_{\mathfrak{d}}+\tau_{3}\left(\delta_{\mathfrak{n}}\right), \frac{2}{\alpha}, 2\right)+\Gamma_{1} e^{-k\left(t-T^{*}\right)}
$$

It is direct to show that for all $e \in \mathbb{R}^{n}$

$$
\frac{\lambda_{m}(S)}{\lambda_{M}(S) \theta^{2(n-1)}} W_{\alpha, \beta}(e) \leq W_{\alpha, \beta}(\bar{e}) \leq \frac{\lambda_{m}(S)}{\lambda_{M}(S)} W_{\alpha, \beta}(e) .
$$

It follows that

$$
\begin{aligned}
& W_{\alpha, \beta}(e(t)) \leq \frac{\lambda_{M}(S)}{\lambda_{m}(S)} \theta^{2(n-1)} \\
& \times\left(\nu\left(4 \frac{\sqrt{\lambda_{M}(S)}}{\theta^{n}} \delta_{\mathfrak{d}}+\tau_{3}\left(\delta_{\mathfrak{n}}\right), \frac{2}{\alpha}, 2\right)+\Gamma_{1} e^{-k\left(t-T^{*}\right)}\right), \\
\leq & \frac{\lambda_{M}(S)}{\lambda_{m}(S)}\left(4 \frac{\sqrt{\lambda_{M}(S)}}{\theta} \delta_{\mathfrak{d}}+\theta^{n-1} \tau_{3}\left(\delta_{\mathfrak{n}}\right)\right)^{2} \\
& +\frac{\lambda_{M}(S)}{\lambda_{m}(S)} \theta^{2(n-1)} \Gamma_{1} e^{-k\left(t-T^{*}\right)}, \\
\triangleq & \left(\frac{c}{\theta} \delta_{\mathfrak{d}}+\theta^{n-1} \tau\left(\delta_{\mathfrak{n}}\right)\right)^{2}+\Gamma e^{-k\left(t-T^{*}\right)},
\end{aligned}
$$

where $c, \Gamma, k>0$ and $\tau$ is a class $\mathcal{K}_{\infty}$ function.

\section{References}

[1] T. Ahmed-Ali, E. Cherrier, and F. Lamnabhi-Lagarrigue. Cascade high-gain predictors for a class of nonlinear systems. IEEE Transactions on Automatic Control, 57(1):221-226, 2012.

[2] V. Andrieu and L. Praly. On the existence of a kazantziskravaris/luenberger observer. SIAM Journal on Control and Optimization, 45(2):432-456, 2006.

[3] V. Andrieu, L. Praly, and A. Astolfi. Homogeneous approximation, recursive observer design, and output feedback. SIAM Journal on Control and Optimization, 47(4):1814-1850, 2008

[4] V. Andrieu, L. Praly, and A. Astolfi. High gain observers with updated gain and homogeneous correction terms. Automatica, 45(2):422-428, 2009.

[5] T.M. Apostol. Mathematical analysis. Addison-Wesley, $2^{\text {nd }}$ edition, 1974.

[6] J. Back, K.T. Yu, and J.H. Seo. Dynamic observer error linearization. Automatica, 42(12):2195-2200, 2006.

[7] S. Battilotti. Incremental generalized homogeneity, observer design and semiglobal stabilization. Asian Journal of Control, 16(2):498-508, 2014.

[8] S. P. Bhat and D. S. Bernstein. Finite-time stability of continuous autonomous systems. SIAM Journal of Control and Optimization, 38(3):751-766, 2000.

[9] S. P. Bhat and D. S. Bernstein. Geometric homogeneity with applications to finite-time stability. Mathematics of Control, Signals and Systems, 17:101-127, 2005.

[10] I. Bouraoui, M. Farza, T. Ménard, R.B. Abdennour, and M. M'Saad H. Mosrati. Observer design for a class of uncertain nonlinear systems with sampled outputsapplication to the estimation of kinetic rates in bioreactors. Automatica, 55:78-87, 2015.

[11] C. Califano and C.H. Moog. The observer error linearization problem via dynamic compensation. IEEE Transactions on A utomatic Control, 59(9):2502-2508, 2014.

[12] G. Conte, C.H. Moog, and A.M Perdon. Algrebraic Methods for Nonlinear Control Systems. Springer, second edition, 2007. 
[13] S. Diop and M. Fliess. Nonlinear observability, identifiability, and persistent trajectories. In Proceedings of the 30th IEEE Conference on Decision and Control, Brighton, England, December 1991.

[14] S. Diop and M. Fliess. On nonlinear observability. In Proc. 1st Europ. Control Conf, pages 152-157, 1991.

[15] F. Esfandiari and H.K. Khalil. Output feedback stabilization of fully linearizable systems. International Journal of control, 56(5):1007-1037, 1992.

[16] M. Farza, M. M'Saad, and L. Rossignol. Observer design for a class of mimo nonlinear systems. Automatica, 40(1):135143, 2004 .

[17] T. Floquet and J.P. Barbot. Super twisting algorithm based step-by-step sliding mode observers for nonlinear systems with unknown inputs. International Journal of Systems Science, 38(10):803-815, 2008.

[18] J.P. Gauthier, H. Hammouri, and S. Othman. A simple observer for nonlinear systems. applications to bioreactors. IEEE Transactions on Automatic Control, 37(6):875-880, 1992.

[19] H. Hammouri, B. Targui, and F. Armanet. High gain observer based on a triangular structure. International journal of Robust and Nonlinear structure, 12(6):497-518, 2002.

[20] R. Hermann and A.J. Krener. Nonlinear controllability and observability. IEEE Transactions on automatic control, 22(5):728-740, 1977.

[21] P. Jouan. Immersion of nonlinear systems into linear systems modulo output injection. SIAM Journal on Control and Optimization, 41(6):1756-1778, 2003.

[22] H.K. Khalil. Nonlinear Systems. Prentice-Hall, 1996.

[23] A.J. Krener and A. Isidori. Linearization by output injection and nonlinear observers. Systems \& Control Letters, 3(1):47$52,1983$.

[24] A.J. Krener and W. Respondek. nonlinear observers with linearizable error dynamics. SIAM Journal of Control and Optimization, 23(2):197-216, 1985.

[25] Y. Li, Y. Shen, and X. Xia. Global finite-time observers for a class of nonlinear systems. Kybernetika, 49(2):319-340, 2013.

[26] Y. Li, X. Xia, and Y. Shen. A high-gain-based global finitetime nonlinear observer. International Journal of Control, 86(5):759-767, 2013.

[27] T. Ménard, E. Moulay, and W. Perruquetti. A global highgain finite-time observer. IEEE Transactions on Automatic Control, 55(6):1500-1506, 2010.

[28] P.H. Menold, R. Findeisen, and F. Allgöwer. finite-time convergent observers for linear time varying systems. In Proceedings of the $11^{\text {th }}$ Mediterranean Conference on Control and Automation, 2003.

[29] H. Michalska and D. Mayne. Moving horizon observers and observer-based control. IEEE Transactions on Automatic Control, 40:995-1006, 1995.

[30] J. Munkres. Topology. Prentice Hall, 2 edition, 1999.

[31] Y. Orlov. Finite time stability and robust control synthesis of uncertain switched systems. SIAM Journal on Control and Optimization, 43(4):1253-1271, 2004.

[32] W. Perruquetti, T. Floquet, and E. Moulay. Finite-time observers: application to secure communication. IEEE Transactions on Automatic Control, 53(1):356-360, 2008.

[33] F. Plestan, J.W. Grizzle, E.R. Westervelt, and G. Abba. Stable walking of a 7-dof biped robot. IEEE Tansactions on Robotics and Automation, 19(4):653-668, 2003.
[34] A. Polyakov. Nonlinear feedback design for fixed-time stabilization of linear control systems. IEEE Transactions on Automatic Control, 57(8):2106-2110, 2012.

[35] A. Polyakov, D. Efimov, and W. Perruquetti. Finite-time and fixed-time stabilization: Implicit lyapunov function approach. Automatica, 51:332-340, 2015.

[36] L. Rosier. Homogeneous lyapunov function for homogeneous continuous vector field. Systems \& Control Letters, 19(6):467-473, 1992.

[37] Y. Shen and Y. Huang. Uniformly observable and globally lipschitzian nonlinear systems admit global finitetime observers. IEEE Transactions on Automatic Control, 54(11):2621-2625, 2009.

[38] Y. Shen and X. Xia. Semi-global finite-time observers for nonlinear systems. Automatica, 44(12):3152-3156, 2008.

[39] Y. Shen and X. Xia. Semi-global finite-time observers for a class of non-lipschitz systems. In $8^{\text {th }}$ IFAC Symposium on Nonlinear Control Systems, pages 421-426, Bologna, Italy, 2010.

[40] S.T. Venkataraman and S. Gulati. Terminal sliding modes: a new approach to nonlinear control synthesis. In $5^{\text {th }} I E E E$ International Conference on Advanced Robotics, pages 443448, 1991. 